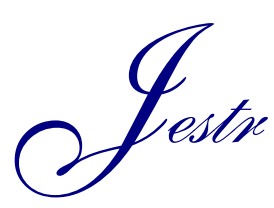

\title{
Study on the Improved Unscented Kalman Filter Ultra-Wideband Indoor Location Algorithm based on Two-Way Time-of-Flight
}

\author{
Feng Tian* and Hanqing Li \\ College of Communication and Information Engineering, Xi'an University of Science and Technology, Xi'an 710054, China
}

Received 4 September 2018; Accepted 23 November 2018

\begin{abstract}
Traditional indoor location technologies based on wireless sensor network have difficulty in satisfying the requirements of positioning accuracy and real-time tracking in the non-line-of-sight (NLOS) environment due to considerable errors. In this study, an indoor location and tracking algorithm for the ultra-wideband (UWB) system was proposed to solve accurate location and tracking problems of the moving target in the complicated indoor environments. First, this algorithm measured the distance between tag and anchor accurately by the two-way time-of-flight (TW-TOF) method, and then calculated the position coordinates of the tag by the CHAN algorithm after the range measurement. Second, the coordinates of tag position were used as the observation value of unscented Kalman filter (UKF) with the state updating equation of UKF modified. Finally, the real-time position states and speed of the tag could be acquired. The proposed algorithm was compared with the extended Kalman filter (EKF) through a simulation experiment. Results demonstrate that the improve UKF algorithm can realize accurate location and dynamic tracking of the moving target in the indoor NLOS environment. The location accuracy of the proposed algorithm is 33.5\% higher than that of the EKF algorithm. The study can provide certain references for accurate location and tracking of the moving target in the complicated indoor environments.
\end{abstract}

Keywords: positioning algorithm, two-way ranging, non-line-of-sight, ultra-wideband, unscented Kalman filter

\section{Introduction}

The wireless communication technology has rapidly progressed as a response to the era of big data. Social demands for location awareness increase gradually to acquire accurate location information of targets and realize real-time tracking. Currently, outdoor location techniques become increasingly mature and are extensively used in all aspects of social life. However, abundant barriers between the target and anchor along with serious non-line-of-sight (NLOS) are present in complicated indoor environments. Thus, using traditional outdoor location techniques is difficult in indoor environments. As a result, indoor location technique is accepted as an important study field. Common indoor location techniques are mainly based on wireless local area network, radio frequency tag, Zigbee technology, Bluetooth technology, ultra-wideband (UWB) and infrared technology. These techniques have achieved abundant results and have thus facilitated the development of indoor location technologies to some extent. The UWB technology is superior to many location technologies due to its high location accuracy, and high temporal resolution, and strong anti-multipath ability, and strong signal penetration, which has achieved considerable development in recent years [1-3].

In wireless indoor location systems, location algorithms are divided into range-based location and range-free location. The former shows higher location accuracy than the latter

*E-mail address: tianfeng0306@163.com

ISSN: $1791-2377$ @ 2018 Eastern Macedonia and Thrace Institute of Technology. All rights reserved. doi:10.25103/jestr.115.11 and covers two steps of range measurement and location. Therefore, the accuracy of range-based location is determined by ranging technology and location algorithm. At present, time of arrival (TOA), received signal strength indicator (RSSI), time difference of arrival (TDOA) and arrival of angle (AOA) are common ranging methods. Two methods can also be combined to complement each other, such as TOA and AOA combined position technology [4]. Location accuracy is sensitive to the spreading of NLOS and multipath effect in complicated environments with abundant barriers between the tag and anchor; thus, the influences of NOLS should be decreased or eliminated urgently [5].

To address the above-mentioned problems, two-way time-of-flight (TW-TOF) method and CHAN algorithm were combined by the UWB location technology in the present study. Moreover, a moving target was located and traced by the improved unscented Kalman filter (UKF) to estimate the location of the moving target accurately and thus provide references for accurate location and tracking of the moving target in the complicated indoor environments.

\section{State of the art}

Considerable studies have been conducted on target location and tracking in complicated indoor environments from aspects of location algorithm [6-7], filtering estimation algorithm [8-9], and reducing effects of NLOS [10]. A Newton location algorithm based on RSSI was proposed by Yangqianzi Luo, which achieved smaller measuring error by 
preprocessing of range based on particle filtering algorithm and estimated coordinates of location node by Newton's method. However, the RSSI method presents large errors [11]. An approximate maximum likelihood estimation method based on the approximate maximum likelihood location fuzzy elimination method was put forward to modify CHAN algorithm by Zijia Wang, with promising engineering application prospect possessed, which, however, neglected the influences of NLOS [12]. An improved constraint weighted least squares algorithm was proposed to solve the anomaly of measurement matrix that might occur in AOA and TDOA combined location by Leibing Yan, which achieved high location accuracy [13]. A location method based on rectangular coordinates and a location method based on polar coordinates by combining TDOA and AOA were propounded by Congfeng Liu, which provided certain theoretical references. Nevertheless, the location method based on AOA requires the corresponding antenna array, which consumes high cost and is difficult to be realized [14]. With the range measured by TOA, the location accuracy further increased by making fully utilizing the nonlinear filtering characteristics of the extended Kalman filter (EKF) algorithm by Fengchong Wang. Nevertheless, the EKF algorithm must remove second-order or higherorder terms in the process of the linearization, which may cause certain errors [15]. The optimal course estimation of the moving target was carried out through the particle filter method by Ling Pei. This approach still has some disadvantages, such as heavy calculation loads and poor performance of real-time location [16]. A new robust second-order cone relaxation (SOCR) method by combining TOA was proposed by Shengjin Zhang, which, however, was insensitive to NLOS errors and presented lower calculation complexity than existing methods. Although this robust SOCR method has certain reference values, it neglects timeliness of location [17]. To address the significantly reduction in estimation accuracy of traditional location algorithms in multipath and NLOS environments, an indoor location algorithm based on AOA and phase difference of arrival was put forward by Yongtao Ma. Subsequently, the actual position of the tag was calculated by combining the weighted least squares algorithm and residual weighted algorithm, which can offer references for reducing the influences of NLOS [18]. A low-complexity NLOS control method based on the sparse pseudo-input Gaussian process was proposed by Yang $\mathrm{X}$, which was considerably faster than the latest advanced machine learning method and gained comparable performances under small pseudo-data size. Nevertheless, the UWB signal characteristics in line-of-sight (LOS)/NLOS environment must be collected in advance, which restricts its practical applications to some extent [19]. On the basis of sampling of UWB signal characteristics in LOS/NLOS environment, a random forest discrimination model was constructed by Ling Zeng to identify NLOS anchors. This model applied different NLOS elimination methods for situations involving different NLOS anchors, thus increasing the location accuracy effectively. However, the range error between a moving target and anchor caused by system noises still must be solved [20].

The above-mentioned studies barely involve the tracking of moving targets. A large improvement space exists in decreasing or eliminating the influences of NLOS, and the location algorithm still can be further optimized. To solve accurate location and tracking of moving targets in complicated indoor environments, advantages and disadvantages of existing algorithms in this field were considered comprehensively in this study. The range between sensor nodes was measured by TW-TOF method, which has no time synchronization between the tag and anchors and can thus avoid the additional cost for acquiring time synchronization. After the range was measured, coordinates of location were calculated preliminarily by the CHAN algorithm and then used as an observation data of UKF with the state updating equation of UKF modified. Therefore, the iteration error was decreased and the location accuracy was increased effectively. The proposed algorithm is applicable to accurate location and dynamic tracking of a moving target in the indoor NLOS environment. The calculation complexity of the proposed algorithm is slightly lower than that of the EKF algorithm and the location performance is approximate to that under LOS state.

The remainder of this study is organized as follows. Section 3 introduces the TW-TOF method, the CHAN algorithm, and the improvement of the UKF algorithm. Section 4 designs a simulation experiment for comparative verification between the proposed algorithm and EKF algorithm. Section 5 elaborates the conclusions.

\section{Methodology}

Asynchronous TW-TOF method is employed to measure the time of flight of UWB impulse accurately for obtaining the distance between the tag and anchor. Thereafter, coordinates of the tag are calculated by CHAN algorithm based on range and used as the observation data of UKF algorithm. Finally, location and tracking of the tag are realized.

\subsection{Two-way time-of-flight}

The asynchronous TW-TOF method requires no time synchronization of two communication parties and thus saves the corresponding cost, which requires the tag and anchor to be able to send and receive signals simultaneously. The tag can initialize range information periodically, while the anchor can receive signals emitted by the tag timely. The distance between the anchor and tag can be calculated in accordance with the difference in time based on data interaction. The measurement steps of TW-TOF method are shown in Fig. 1.

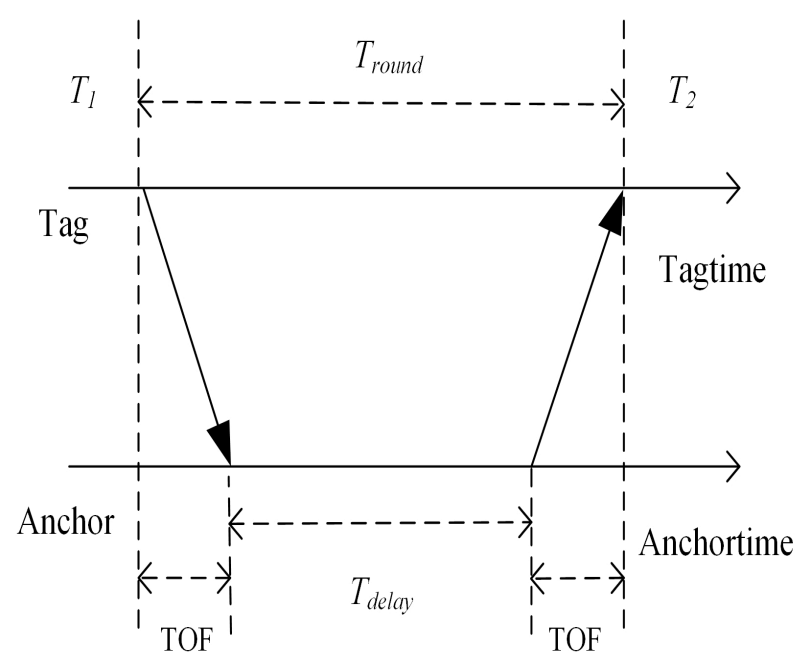

Fig. 1. Measurement steps of the TW-TOF method 
The tag sends information to the anchor and record the transmission time (namely, the send timestamp) ( $\left.T_{1}\right)$. The anchor receives information and responds to the tag after a certain delay. Thereafter, the tag receives the response and records the received time stamp $\left(T_{2}\right)$.

The total round time $\left(T_{\text {round }}\right)$ can be gained from $T_{1}$ and $T_{2}$ TOF value $(t)$ can be acquired by setting the fixed delay of time ( $\left.T_{\text {delay }}\right)$ for the anchor to send the signals:

$$
t=\frac{T_{2}-T_{1}-T_{\text {delay }}}{2}
$$

On this basis, the range $r$ can be acquired as follows:

$$
r=\frac{c\left(T_{2}-T_{1}-T_{\text {delay }}\right)}{2}
$$

where $c$ is the signal velocity, that is, velocity of light.

\subsection{CHAN algorithm}

The distances of the tag to at least three anchors can be acquired by the two-way TOA location method based on the time of flight. Subsequently, location of the tag is calculated by the CHAN algorithm to gain the coordinates of the tag to be located. The CHAN algorithm is solved using the least squares algorithm twice to obtain non-iterative closed-form solution to the corresponding nonlinear equation set. When the range error of the location system obeys Gaussian distribution, the CHAN algorithm presents high location accuracy. The solving process of CHAN algorithm is introduced as follows.

$T(x, y)$ is set as the tag (namely, the estimating position) and $X_{i}\left(x_{i}, y_{i}\right)$ represents the coordinates of the anchor. $n$ is the number of known anchors. The distance $r_{i}$ between the tag and the anchor $X_{i}$ is:

$$
r_{i}=\sqrt{\left(x_{i}-x\right)^{2}+\left(y_{i}-y\right)^{2}}
$$

The anchor $X_{i}$ is used as the standard. The range difference $r_{i, 1}$ of the tag $T$ to $X_{i}(i \neq 1)$ and $X_{1}$ is:

$$
r_{i, 1}=c t_{i, 1}=r_{i}-r_{1}, i=1,2, \ldots n
$$

where $c$ is the velocity of light, and $t_{i, 1}$ is the difference of time of the tag $T$ to $X_{i}(i \neq 1)$ and $X_{1}$. From Eqs. (3) and (4), the following condition can be obtained:

$$
x_{i, 1}+y_{i, 1} y+r_{i, 1} r_{1}=\frac{1}{2}\left(K_{i}-K_{1}-r_{i, 1}^{2}\right)
$$

where $K_{i}=x_{i}^{2}+y_{i}^{2}, x_{i, 1}=x_{i}-x_{1}$ and $y_{i, 1}=y_{i}-y_{1}$.

$x, y, r_{1}$ are the independent variables and Eq. (5) can be converted into a linear equation set:

$$
G_{a} z_{a}=h
$$

where $\quad G_{a}=-\left(\begin{array}{ccc}x_{2,1} & y_{2,1} & r_{2,1} \\ x_{3,1} & y_{3,1} & r_{3,1} \\ & \cdots & \\ x_{n, 1} & y_{n, 1} & r_{n, 1}\end{array}\right) \quad, \quad z_{a}=\left(x, y, r_{1}\right)^{T} \quad$ and $h=\frac{1}{2}\left(\begin{array}{c}r_{2,1}^{2}-K_{2}+K_{1} \\ r_{3,1}^{2}-K_{3}+K_{1} \\ \cdots \\ r_{n, 1}^{2}-K_{n}+K_{1}\end{array}\right)$.

$\{*\}^{0}$ is defined as the value without noise. Thus, the error vector in Eq. (6) is:

$e=h-G_{a} Z_{a}^{0} h$

$e$ is assumed to approximately obey Gaussian distribution with a covariance matrix. Therefore, the covariance matrix is:

$\psi=E\left(e e^{T}\right)=c^{2} B Q B$

where $B=\operatorname{diag}\left\{r_{2}^{0}, r_{3}^{0}, \ldots, r_{n}^{0}\right\}$ and $Q$ is the covariance matrix of noise vectors that obeys Gaussian distribution.

The least squares solution of Eq. (6) is equivalent to solving the normal equation.

$$
G_{a}^{T} G_{a} z_{a}=G_{a}^{T} h
$$

Elements in $z_{a}$ are assumed to be independent mutually. The problem turns into a weighted least squares problem after errors of each data group are weighted. Thus, Eq. (7) can be rewritten as follows:

$$
\left(G_{a}^{T} \psi G_{a}\right) z_{a}=G_{a}^{T} \psi h
$$

Therefore, the weighted least squares estimation of $z_{a}$ is:

$$
z_{a}=\left(G_{a}^{T} \psi^{-1} G_{a}\right)^{-1} G_{a}^{T} \psi^{-1} h
$$

$B$ covers the real distance from $T$ to $X_{i}$ and is unknown at calculation. When the distance from $T$ to $X_{i}$ is large, $\Psi$ can be replaced by $Q$. Therefore,

$$
z_{a} \approx \widetilde{z}_{a}=\left(G_{a}^{T} Q^{-1} G_{a}\right)^{-1} G_{a}^{T} Q^{-1} h
$$

The initial solution is obtain from Eq. (12) and is then used to recalculate $B$. Calculate results of $B$ are introduced into Eq. (8) and then to Eq. (10), for obtaining the results of $z_{a}$, which are the first estimation results.

On the basis of the first estimation results, a group of error equation set is reconstructed for the second estimation:

$$
\left\{\begin{array}{l}
z_{a, 1}=x^{0}+e_{1} \\
z_{a, 2}=y^{0}+e_{2} \\
z_{a, 3}=r^{0}+e_{3}
\end{array}\right.
$$


where $z_{a, i}$ denotes the component $i$ of $\mathrm{z}_{a}, i \in[1,3]$. $e_{1}, e_{2}, e_{3}$ are estimation errors of $\mathrm{z}_{a}$.

Therefore, the second estimation is:

$z_{a 1}=\left(G_{a 1}^{T} \psi_{1}^{-1} G_{a 1}\right)^{-1} G_{a 1}^{T} \psi_{1}^{-1} h_{1}$

where $\quad z_{a 1}=\left(\begin{array}{c}\left(x-x_{1}\right)^{2} \\ \left(y-y_{1}\right)^{2}\end{array}\right) \quad, \quad G_{a 1}=\left(\begin{array}{ll}1 & 0 \\ 0 & 1 \\ 1 & 1\end{array}\right)$, $h_{1}=\left(\begin{array}{c}\left(z_{a, 1}-x_{1}\right)^{2} \\ \left(z_{a, 2}-y_{2}\right)^{2} \\ \left(z_{a, 3}\right)^{2}\end{array}\right) \quad, \quad \psi_{1}=4 B_{1} \operatorname{cov}\left(z_{a}\right) B_{1}$ $B_{1}=\operatorname{diag}\left\{x^{0}-x_{1}, y^{0}-y_{1}, r_{1}^{0}\right\}$ and $\operatorname{cov}\left(z_{a}\right)=\left(G_{a}^{0 T} \psi^{-1} G_{a}^{0}\right)^{-1}$. The pre-estimation results of tag coordinates are:

$$
(x, y)^{T}= \pm \sqrt{z_{a_{1}}}+\left(x_{1}, y_{1}\right)^{T}
$$

\subsection{Kalman filter algorithm}

The combined TW-TOF and CHAN algorithm mainly calculates coordinates of a static tag. The location methods for the static target cannot be applied to the moving target directly because the coordinates at the current and previous moments are connected to some extent. In this study, the tag is located and tracked by the UKF algorithm with considerations to the possible movements of the tag.

UKF based on unscented transformation (UT) is a nonlinear filter algorithm, which processes nonlinear transmission of mean and covariance through UT and applies the nonlinear model of the system. Thus, UKF realizes the linear processing of nonlinear functions. EKF algorithm is an approximation of the Gaussian distribution of the nonlinear function and implements the system linearization close to the working point. Given the approximate linearization, noise and state are assumed to obey Gaussian distribution. Thus, only the mean and covariance matrix need to be calculated. However, the Jacobian matrix of EKF is relatively complicated. The initial state and covariance are difficult to be determined. Therefore, EKF algorithm is inapplicable to strongly nonlinear systems.

UKF algorithm hypothesizes that the state meets the Gaussian distribution, and the Sigma points are produced through UT to approximate nonlinear mean and variance, thus obtaining a large number of observation defaults, which avoids ignorance of high-order problems in the linearization and only processes the posterior probability density. Therefore, the UKF algorithm obtains the secondary optimal solution and shows higher calculation accuracy and stronger adaptation than the EKF algorithm. In consideration of the discrete time nonlinear system in the NLOS environment, the state and observation equations of UKF are:

$$
\begin{aligned}
& x_{k+1}=f_{k}\left(x_{k}\right)+w_{k} \\
& z_{k}=h_{k}\left(x_{k}\right)+v_{k}+n_{k}
\end{aligned}
$$

where $k \in N$ is the time variable. $x_{k} \in R_{n}$ is the system state vector at $k . x_{k+1} \in R_{n}$ is the system state vector at $k+1$. $n_{k}$ is the practical NLOS error $\left(n_{k}>0\right) . z_{k} \in R_{m}$ is an observation vector at $k \cdot f_{k}$ denotes the propagation function of an n-dimensional state. $h_{k}$ is a measurement function of a m-dimensional vector. $w_{k}$ and $v_{k}$ are independent Gaussian white noises with zero means.

UT, which is a nonlinear approximation method, constructs a group of sampling points (Sigma points) based on mean and variance of current state. And the mean and variance of the nonlinear transformation can be approximated by the mean and variance of this group of sampling points. Given Gaussian white noises, UKF implements the nonlinear functional processing to a few Sigma points and can calculate the statistical characteristics of random variables.

The statistical characteristic of $x$ is $\left(\bar{x}, P_{x}\right)$ and a total of $2 n+1$ Sigma points are constructed. If $\xi_{i}(i=0,1,2 \cdots, 2 n)$, then the method to produce Sigma points is:

$\left\{\begin{array}{l}\xi_{0}=\bar{x} \\ \xi_{0}=\bar{x}+\left(\sqrt{(n+\lambda) P_{x}}\right)_{i}, i=1,2, \ldots, n \\ \xi_{0}=\bar{x}+\left(\sqrt{(n+\lambda) P_{x}}\right)_{i}, i=n+1, n+2, \ldots, 2 n\end{array}\right.$

where $\lambda=\alpha^{2}(n+k)-n$ is a proportional coefficient, $n$ is the dimension of state, and $\alpha$ determines the scattering degree of Sigma (generally 0.01 ). Influences of high-order terms are minimized by adjusting the value of $\alpha . k$ is an adjustable parameter that has a value of 0 in general. $\left(\sqrt{(n+\lambda) P_{x}}\right)_{i}$ is the column $i$ of the new matrix gained after square root matrix is collected from the matrix $(n+\lambda) P_{x}$.

UKF algorithm is mainly divided into state prediction, observation prediction and state updating. A group of sampling points (Sigma points) and the corresponding weights are obtained through UT. The one-step prediction values of the group of sampling points are calculated for calculating the one-step prediction and covariance matrix of the state variable. In accordance with the one-step prediction value, UT is used again to produce a new Sigma point set. This set is introduced into the observation equation to predict observation variables and update the state.

The state prediction equation is:

$$
\left\{\begin{array}{l}
\xi_{k \mid k-1}^{(i)}=f_{k}\left(\xi_{k-1 \mid k-1}^{(i)}\right), i=0,1, \ldots, 2 n \\
\hat{x}_{k \mid k-1}=\sum_{i=0}^{2 n} \omega_{l}^{(m)} \xi_{k \mid k-1}^{(i)} \\
P_{k \mid k-1}=\sum_{i=0}^{2 n} \omega_{l}^{(c)}\left(\xi_{k \mid k-1}^{(i)}-\hat{x}_{k \mid k-1}\right)\left(\xi_{k \mid k-1}^{(i)}-\hat{x}_{k \mid k-1}\right)^{T}+Q_{k-1}
\end{array}\right.
$$

where $Q_{k}$ is the variance of $w_{k}$ and $\omega$ is the corresponding weight.

The prediction equation of observation variables is: 


$$
\left\{\begin{array}{l}
\zeta_{k \mid k-1}^{(i)}=h_{k}\left(\xi_{k}^{(i)}\right), i=0,1, \cdots, 2 n \\
\hat{z}_{k \mid k-1}=\sum_{i=0}^{2 n} \omega_{i}^{(m)} \zeta_{k \mid k-1}^{(i)} \\
P_{x_{k} z_{k}}=\sum_{i=0}^{2 n} \omega_{i}^{(c)}\left(\xi_{k \mid k-1}^{(i)}-\hat{x}_{k \mid k-1}\right)\left(\zeta_{k \mid k-1}^{(i)}-\hat{z}_{k \mid k-1}\right)^{T} \\
P_{z_{k}}=\sum_{i=0}^{2 n} \omega_{i}^{(c)}\left(\zeta_{k \mid k-1}^{(i)}-\hat{z}_{k \mid k-1}\right)\left(\zeta_{k \mid k-1}^{(i)}-\hat{z}_{k \mid k-1}\right)^{T}+R_{k}
\end{array}\right.
$$

where $R_{k}$ is the variance corresponding to $v_{k}$.

The state updating equation is:

$$
\left\{\begin{array}{l}
\hat{x}_{k \mid k}=\hat{x}_{k \mid k-1}+K_{k}\left(z_{k}-\hat{z}_{k \mid k-1}\right) \\
K_{k}=P_{x_{k} z_{k}} P_{z_{k}}^{-1} \\
P_{k \mid k}=P_{k \mid k-1}-K_{k} P_{z_{k}} K_{k}^{T}
\end{array}\right.
$$

NLOS errors can obey Delta distribution, uniform distribution or exponential distribution in different channel environments. In this study, NLOS errors are assumed to obey the exponential distribution. $x$ is a random variable and conforms to the exponential distribution with parameter $y$. The conditional probability density function is:

$$
f_{x / y}(x / y)=\left\{\begin{array}{l}
\frac{1}{y} \exp \left(-\frac{x}{y}\right) \ldots \ldots \ldots \tau>0 \\
0 \ldots \ldots \ldots \ldots \ldots \ldots \ldots \ldots \ldots \ldots \ldots \ldots \ldots \ldots \ldots \\
\ldots
\end{array}\right.
$$

where $y=T_{1} d \xi$ denotes the root-mean-square delay. $\xi$ reflects the random variable conforming to the logarithmic normal distribution. $d$ is the distance between the tag and anchors. $T_{i}$ is the mid-value of delay extension when the distance is $d$.

The mean and variance of additional delay caused by NLOS propagation can be expressed as follows:

$$
\begin{aligned}
& E\left(t_{i}, N\right)=T_{i} d_{i}^{\varepsilon} e^{\left(m_{z}+\delta_{z}^{2} / 2\right)} \\
& D\left(t_{i}, N\right)=\left(T_{i} d_{i}^{\varepsilon}\right)^{2}\left(e^{m_{z}+\delta_{z}^{2} / 2}\right)^{2}\left(2 e^{\delta_{z}^{2}}-1\right)
\end{aligned}
$$

where $m_{z}$ and $\delta_{z}$ are mean and standard deviation of the Gaussian random variable $z=10 \log \varepsilon . \varepsilon$ is an index between 0.5 and 1 .

In practical applications, the real distance between the tag and unknown nodes is unknown and replaced by the predicted value. Thus, the error caused by NLOS propagation can be obtained as follows:

$$
n_{k}=E\left(t_{i}, N\right) c=\frac{\hat{E}\left(t_{i}, N\right)}{a} c
$$

where $\hat{E}\left(t_{i}, N\right)$ is the mean delay of prediction results and $c$ is the velocity of light. $a$ is a constant. The prediction value is often higher than the real value due to errors. As a result, $a$ is higher than 1 .

If the environment for signal propagation is fixed, then the mean and variance of root-mean-square delay caused by NLOS can only be determined by the distance from the tag to anchors. Thus, the state updating formula of the UKF is improved to eliminate the NLOS errors conforming to the exponential distribution. In this way, the iteration is corrected to reduce iteration error every time:

$$
\hat{x}_{k \mid k}=\hat{x}_{k \mid k-1}+K_{k}\left(z_{k}-\hat{z}_{k \mid k-1}-n_{k}\right)
$$

Combined with the initial coordinates of the tag obtained by the CHAN algorithm, the UKF algorithm is improved in accordance with the characteristics of the NLOS environment, which can decrease NLOS errors effectively and obtain estimation results close to those in the LOS environment. As a result, accuracy of location and tracking can be guaranteed.

\section{Result Analysis and Discussion}

To verify the validity of the proposed algorithm, the tag was located and traced accurately in the Matlab environment. The results were compared with those of the EKF algorithm. Three anchors with coordinates of $(0 \mathrm{~m}, 0 \mathrm{~m}),(110 \mathrm{~m}, 190$ $\mathrm{m})$, and $(220 \mathrm{~m}, 0 \mathrm{~m})$ were set in an equilateral triangle. The real coordinates of the tag were $\mathrm{X}(160 \mathrm{~m}, 0 \mathrm{~m})$. Distances from the tag to every anchor were acquired by the TW-TOF ranging model. Thereafter, the initial coordinates of unknown nodes were calculated as $\mathrm{X} 0(148.6 \mathrm{~m},-3.7 \mathrm{~m})$ by the CHAN algorithm, which was used as the initial value of UKF algorithm. Figs. 2 and 3 show the state trails of the tag in the $\mathrm{X}$ and $\mathrm{Y}$ directions, respectively.

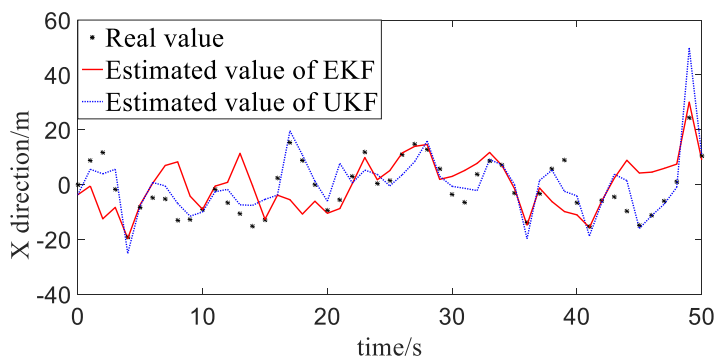

Fig. 2. State trail of $\mathrm{X}$ direction

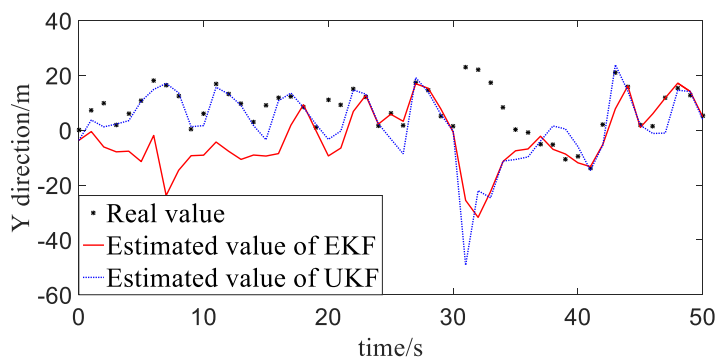

Fig. 3. State trail of $Y$ direction

The state trails of the tag with time by the UKF and EKF algorithms were compared (Fig. 2 and Fig. 3). The coordinates estimated by the EKF algorithm are considerably higher than the real values. 


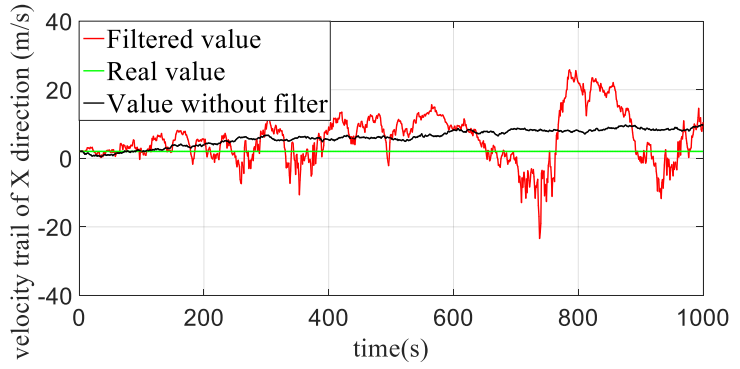

Fig. 4. Velocity trail of $\mathrm{X}$ direction

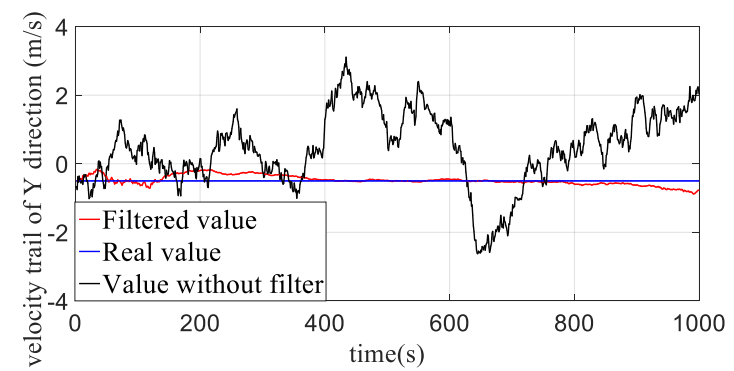

Fig. 5. Velocity trail of $\mathrm{Y}$ direction

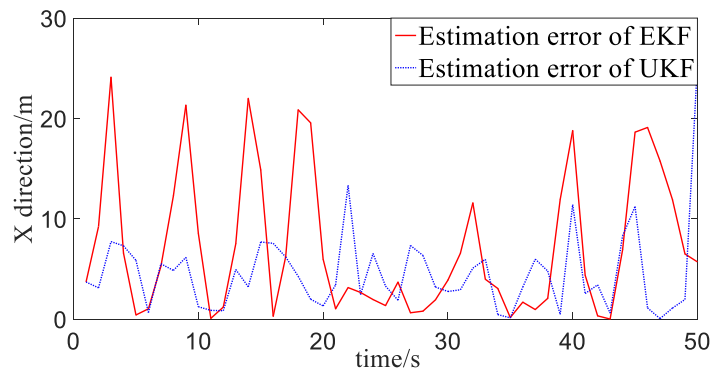

Fig. 6. Estimation error along the $\mathrm{X}$ direction

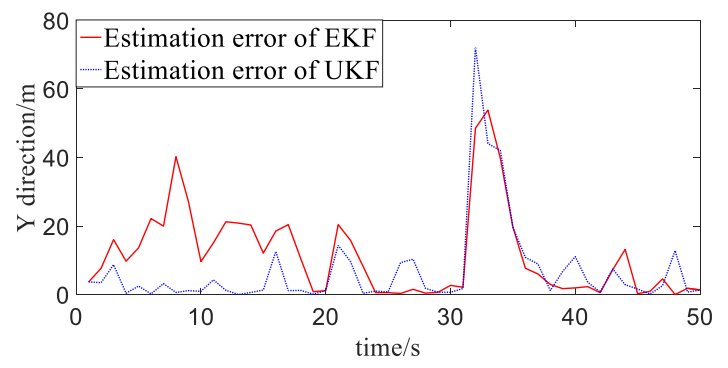

Fig. 7. Estimation error along the Y direction

Figs. 4 and 5 show the velocity trails of the tag along the $\mathrm{X}$ and $\mathrm{Y}$ directions, respectively. The filtered velocity trails are close to the real value. Figs. 6 and 7 show the location errors of EKF and UFK algorithms along the $\mathrm{X}$ and $\mathrm{Y}$ directions, respectively. The location error of UKF algorithm is considerably smaller than that of the EKF algorithm and the location accuracy of the former is $33.5 \%$ higher than that of the latter. The EFK algorithm omits second-order and higher-order terms during the approximate linearization of the system. Moreover, linear working point is only an estimated mean rather than the real mean of input state in most cases, thereby showing great accuracy loss. On the contrary, the UKF algorithm dose not implement linear approximation, but employs Gaussian approximation after the nonlinear transformation of the sigma point. Accordingly, the resulting error is small.

\section{Conclusions}

The complicated indoor NLOS environment can influence location considerably. Traditional location technologies have difficulty in realizing accurate location and tracking of the moving target. To relieve the influences of NLOS and increase the location accuracy of the moving target, an indoor location and tracking algorithm applicable to the UWB system was proposed, which combined the TW-TOF and CHAN algorithms and employed the improved UKF algorithm for location and tracking of the moving target. From the results, the conclusions could be drawn as follows:

(1) The combination of TW-TOF and CHAN algorithms can achieve relatively good preliminary location results. TW-TOF can avoid time synchronization problem and has high ranging accuracy. The CHAN algorithm, which employs the weighted least square algorithm twice, can use range information reasonably and achieve a balance between complexity and accuracy.

(2) The improved UKF algorithm reduces the iteration error in the state updating process and shows high location and tracking accuracy of the moving target in the NLOS environment. The improved UKF algorithm achieves considerably higher location and tracking accuracy than the EKF algorithm.

The proposed indoor location and tracking algorithm not only can fully utilize the high time resolution of UWB but also can offset poor location accuracy of traditional technologies (e.g., WIFI and Bluetooth) to the nonlinear moving target in the indoor NLOS environment. The proposed algorithm, which has good filtering, location and tracking performances, provides some references for wireless indoor location scenes with high requirements on location accuracy. Although the TW-TOF algorithm can use the advantages of UWB technology effectively, the combination of several location means is superior and thus increases the location accuracy. Meanwhile, location and tracking of multiple targets should be considered in future studies.

\section{Acknowledgements}

This work was supported by the Project of Science and Technology of Shaanxi (No.2018GY-151).

This is an Open Access article distributed under the terms of the Creative Commons Attribution License

\section{References}

1. Yin, Z., Jiang, X., Yang, Z., et al., "WUB-IP: A High-Precision UWB Positioning Scheme for Indoor Multiuser Applications". IEEE Systems Journal, 2018, doi:10.1109/JSYST.2017.2766690.
2. Enrique García, Pablo Poudereux, Álvaro Hernández, Juan Jesús García, Jesús Urena., "DS-UWB-indoor-positioning-systemimplementation". Sensors and Actuators, v201, 2013, pp. 172-181 
3. Zhang, J., Shen, C., "Research on UWB Indoor Positioning Based on Improved TDOA Algorithm and Kalman Filter". Modern Electronics Technique, 39(13), 2016, pp. 1-5.

4. Chen, X., Wang, D., Liu, R., Yin, J., Wu, Y., "Structural total least squares algorithm for locating multiple disjoint sources based on AOA/TOA/FOA in the presence of system error". Frontiers of Information Technology \&amp; Electronic Engineering, 19(07), 2018, pp. 917-936.

5. Li, S., Hua, J., Wang, D., Zhou, K., Chen, F., Yu, X., “A Lagrangian Multiplier Improved Constrained Least Squares Localization Algorithm in NLOS Environment". Chinese Journal of Sensors and Actuators, 31(08), 2018, pp. 1235-1239.

6. Guo, K., "Research and application based on TDOA and AOA positioning system”. Digital Technology and Application, 2017(08), 2017, pp. 78-80+84.

7. Dai, Z., Li, X., Chen, Z., He, X., "Research on variable weight indoor fingerprint localization algorithm based on KNN algorithm". Computer Engineering, 2018, doi:10.19678/j.issn.10003428.0050886

8. Seungwon Oh, Minsoo Hahn, Jinsul Kim., "Dynamic EKF-based SLAM for autonomous mobile convergence platforms". Multimedia Tools and Applications, 74(16), 2015, pp. 6413-6430.

9. Meenakshi Gupta, Laxmidhar Behera, Venkatesh K. Subramanian, Mo M. Jamshidi., "A Robust Visual Human Detection Approach With UKF-Based Motion Tracking for a Mobile Robot". IEEE Systems Journal, 9(4), 2015, pp. 1363 - 1375.

10. Reza Monir Vaghefi, R. Michael Buehrer., "Cooperative Localization in NLOS Environments Using Semidefinite Programming". IEEE Communications Letters, 19(8), 2015, pp. $1382-1385$.

11. Luo, Y., Liao, W., "Newton Localization Algorithm Based on Particle Filter for RSSI Ranging Optimization". Instrument Technique and Sensor, 2017(06), 2017, pp. 116-119+124.
12. Wang, Z., Yu, H., Hu, Y., "Improved CHAN algorithm based on maximum likelihood criterion". Computer Applications and Software, 31(09), 2014, pp. 240-243.

13. Yan, L., Lu, Y., Zhang, Y., "TDOA/AOA positioning method based on improved least squares algorithm". Chinese Journal of Radio Science, 31(02), 2016, pp. 394-400.

14. Liu, C., Yang, J., Wang, F., "Joint TDOA and AOA location algorithm". Journal of Systems Engineering and Electronics, 24(2), 2013, pp. $183-188$

15. Wang, F., Zhou, J., Lu, C., An, S., Xiao, W., Lin, B., "Research on indoor positioning of UWB UAV based on EKF filtering". Practical Electronics, 2018(16), 2018, pp.15-16.

16. Pei, L., Liu, D., Zou, D., Ronald Lee Fook Choy, Chen, Y., He, Z., "Optimal Heading Estimation Based Multidimensional Particle Filter for Pedestrian Indoor Positioning”. IEEE Access, 2018(6), 2018, pp. 49705-49720.

17. Zhang, S., Gao, S., Wang, G., Li, Y., "Robust NLOS Error Mitigation Method for TOA-Based Localization via Second-Order Cone Relaxation”. IEEE Communications Letters, 19(12), 2015, pp. $2210-2213$

18. Ma, Y., Wang, B., Pei, S., Zhang, Y., Zhang, S., Yu, J., "An Indoor Localization Method Based on AOA and PDOA Using Virtual Stations in Multipath and NLOS Environments for Passive UHF RFID”. IEEE Access, 2018(6), 2018, pp. 31772 - 31782

19. Yang, X., "NLOS Mitigation for UWB Localization Based on Sparse Pseudo-input Gaussian Process". IEEE Sensors Journal, 18(10), 2018, pp. 4311-4316.

20. Zeng, L., Peng, C., Liu, H., "Ultrawideband indoor positioning algorithm based on NLOS identification". Journal of Computer Applications, 38(S1), 2018, pp. 131-134+139. 